\title{
Symptomatic Sinus Bradycardia in a Patient with Solitary Fibrous Tumor/Hemangiopericytoma Treated with Pazopanib
}

\author{
Wataru Shioyama ${ }^{1}$, Toru Oka ${ }^{1}$, Risa Kamada ${ }^{1}$, Toshinari Yagi ${ }^{2}$, Katsuhiko Nakanishi ${ }^{3}$, \\ Tatsuya Nishikawa ${ }^{1}$, Taku Yasui ${ }^{1}$ and Masashi Fujita ${ }^{1}$
}

\begin{abstract}
:
Pazopanib, a multi-targeted tyrosine kinase inhibitor, is associated with cardiovascular adverse events, such as hypertension, cardiac dysfunction, and thromboembolism. However, symptomatic pazopanib-related bradycardia is uncommon. We herein report a case of symptomatic bradycardia of 35 beats per minute in a patient with solitary fibrous tumor/hemangiopericytoma (SFT/HPC) treated with pazopanib for 1 month. His heart rate recovered to a normal range soon after pazopanib cessation. He restarted pazopanib at a reduced dose, which was continued without SFT/HPC progression or bradycardia recurrence. This case highlights the possibility of bradycardia induced by pazopanib and the importance of monitoring the patient's heart rate.
\end{abstract}

Key words: pazopanib, bradycardia, cardiotoxicity, adverse event, solitary fibrous tumor/hemangiopericytoma

(Intern Med Advance Publication)

(DOI: 10.2169/internalmedicine.5347-20)

\section{Introduction}

Pazopanib is a potent multi-target tyrosine kinase inhibitor (TKI) used to treat patients with renal cell carcinoma and solitary fibrous tumor/hemangiopericytoma (SFT/HPC) because it inhibits angiogenesis $(1,2)$. The most commonly reported cardiovascular adverse events of pazopanib include hypertension, cardiac dysfunction, and thromboembolism, all of which are considered severe cardiac adverse events (3). Asymptomatic bradycardia has been reported to occur in $<5 \%$ of cases with the package insert of VOTRIENT $^{\circledR}$, whereas symptomatic bradycardia has not been reported.

We herein report a case of severe symptomatic bradycardia in a patient with SFT/HPC who was treated with pazopanib.

\section{Case Report}

A 49-year-old man without a history of cardiovascular disease or any risk factors was diagnosed with SFT/HPC af- ter resection of a brain tumor in 2010. He was administered additional radiation therapy (60 Gy) to the brain after the operation (Fig. 1). In 2015, he experienced post-operative recurrences of SFT/HPC in the brain, which were treated by gamma knife radiosurgery (GKRS). In 2018, he presented with hip pain, which led to the detection of bone metastases by computed tomography (CT). CT also detected metastases in his lung and liver; however, he was not administered palliative chemotherapy because of the slow progression of the tumors. After that, follow-up continued with regular magnetic resonance imaging (MRI).

In August 2019, the metastases had obviously progressed (Fig. 2) and pazopanib $800 \mathrm{mg}$ daily was initiated. In September 2019, 1 month after pazopanib initiation, he presented with general fatigue, lightheadedness, and sleepiness; however, he did not experience syncope. He was diagnosed with severe bradycardia, with a heart rate of 35 beats per minute (bpm). His blood pressure at admission was 108/61 $\mathrm{mmHg}$, which was not markedly different from his usual blood pressure, his body temperature was $36.3^{\circ} \mathrm{C}$, and his oxygen saturation was $98 \%$ on room air. A laboratory ex-

${ }^{1}$ Department of Onco-Cardiology, Osaka International Cancer Institute, Japan, ${ }^{2}$ Department of Cancer Chemotherapy Center for Outpatients, Osaka International Cancer Institute, Japan and ${ }^{3}$ Department of Neurosurgery, Osaka International Cancer Institute, Japan 


\section{(A)}

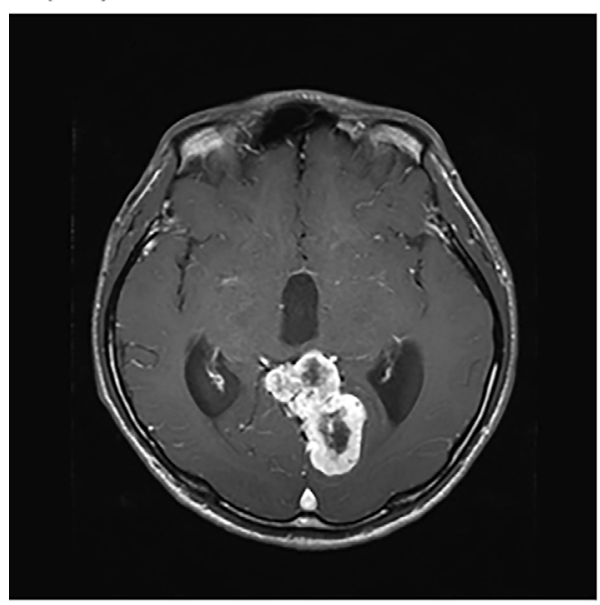

(C)

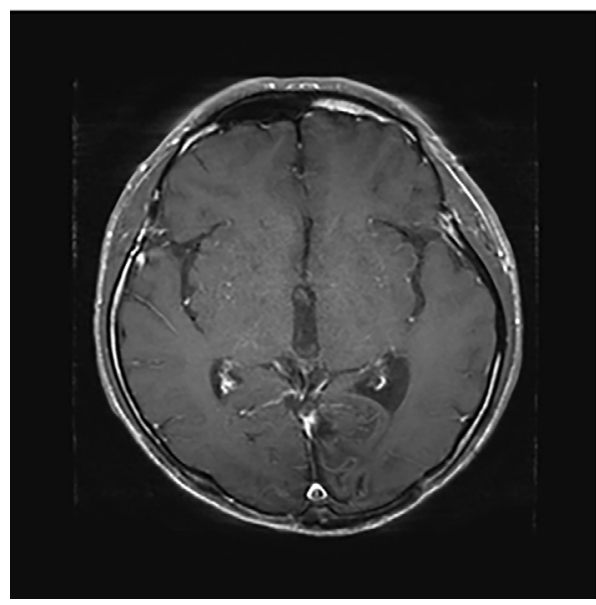

(B)

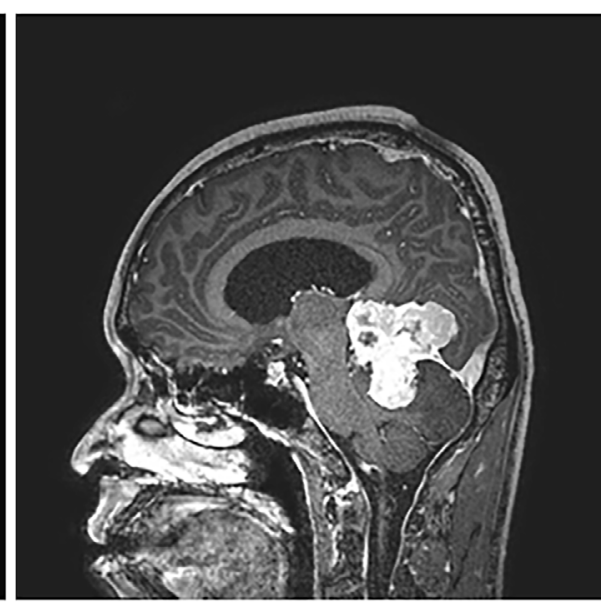

(D)

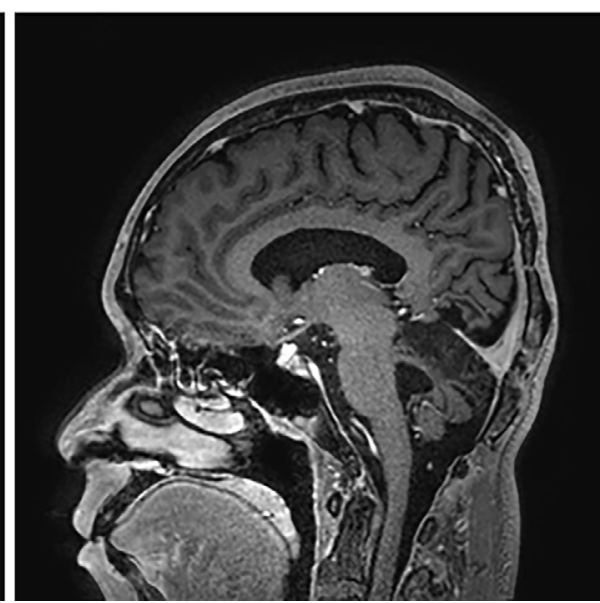

Figure 1. (A, B) Magnetic resonance imaging (MRI) before surgery. Axial (A) and sagittal (B) contrast-enhanced T1-weighted images reveal a heterogeneously enhanced tumor in the left occipital lobe and cerebellum. (C, D) Follow-up MRI after surgery and radiation therapy. Axial (C) and sagittal (D) contrast-enhanced T1-weighted images showed no evidence of a residual lesion.

amination revealed mostly normal findings: white blood cells $4,450 / \mu \mathrm{L}$, hemoglobin $12.3 \mathrm{~g} / \mathrm{dL}$, platelets $9.3 \times 10^{4} / \mu \mathrm{L}$, serum sodium $139 \mathrm{mEq} / \mathrm{L}$, serum potassium $4.5 \mathrm{mEq} / \mathrm{L}$, serum calcium $8.4 \mathrm{mg} / \mathrm{dL}$, serum phosphate $4.0 \mathrm{mg} / \mathrm{dL}$, free triiodothyronine $2.4 \mathrm{pg} / \mathrm{mL}$ (reference range [RR]: 1.9-3.2 $\mathrm{pg} / \mathrm{mL}$ ), free thyroxine $0.8 \mathrm{ng} / \mathrm{dL}$ (RR: 0.7-1.5 ng/dL), thyroid-stimulating hormone $6.63 \mu \mathrm{IU} / \mathrm{mL}$ (RR: $0.35-4.94$ $\mu \mathrm{IU} / \mathrm{mL}), \mathrm{N}$-terminal-pro-brain natriuretic peptide $134 \mathrm{pg} /$ $\mathrm{mL}$ (RR: $\leq 125 \mathrm{pg} / \mathrm{mL}$ ), and troponin $\mathrm{I} \leq 0.01 \mathrm{ng} / \mathrm{mL}$ (RR: $\leq 0.026 \mathrm{ng} / \mathrm{mL}$ ). A transthoracic echocardiogram revealed a normal left ventricular ejection fraction of $58 \%$, normal left ventricle size, and no valvular abnormalities. An electrocardiogram (ECG) before pazopanib treatment showed a heart rate of approximately $50 \mathrm{bpm}$ (Fig. 3A). He therefore had slight bradycardia before treatment with pazopanib, but no history of regular vigorous exercise. Severe sinus bradycardia of $34 \mathrm{bpm}$, a normal QRS complex, and a normal QTc of $403 \mathrm{~ms}$ were observed at admission (Fig. 3B). ECG monitoring revealed that the patient's heart rate tended to increase during body movement (Fig. 4) As this was one month into treatment, we considered that the patient had pazopanib-induced symptomatic bradycardia. Sinus bradycardia is defined as a heart rate $<60 \mathrm{bpm}$ in the Common Terminology Criteria for Adverse Events version 5.0 (4), and $34 \mathrm{bpm}$ indicates grade 3 bradycardia. Pazopanib was immediately discontinued, and his heart rate was observed by 24-h ECG monitoring. Three days after pazopanib discontinuation, the patient's heart rate improved to approximately $50 \mathrm{bpm}$ (Fig. 3C), and his general fatigue improved within 5 days.

Because of its effects on the progression of metastatic SFT/HPC, pazopanib was considered indispensable and restarted at a reduced dose of $200 \mathrm{mg} /$ day in an outpatient setting. After 6 months of treatment with the reduced dose of 
(A)

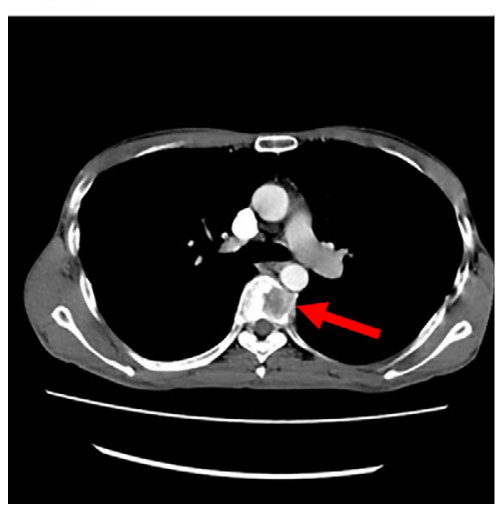

(B)

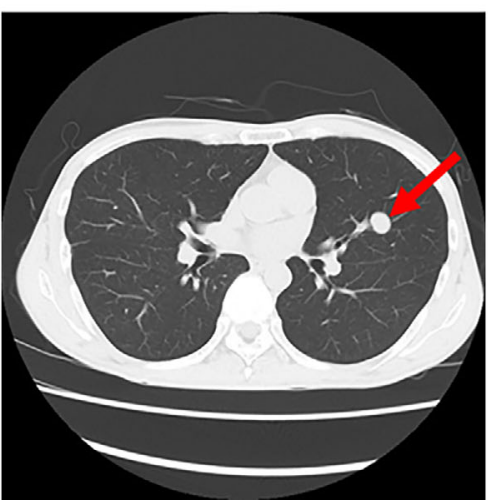

(C)

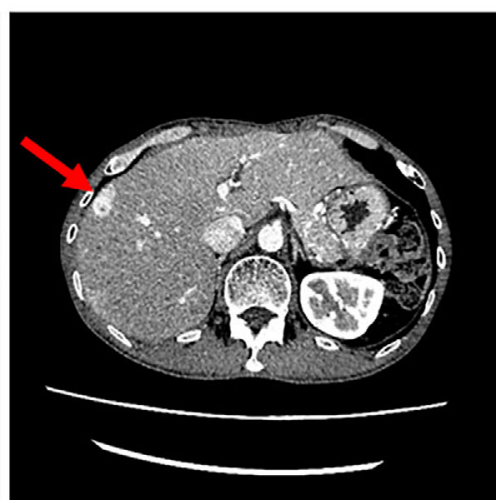

Figure 2. Contrast-enhanced computed tomography shows multiple metastases (arrows) in the thoracic spine (A), lung (B), and liver $(C)$.

(A)

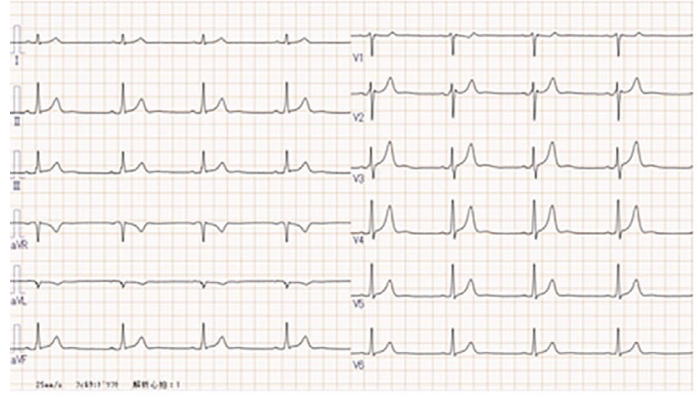

(C)

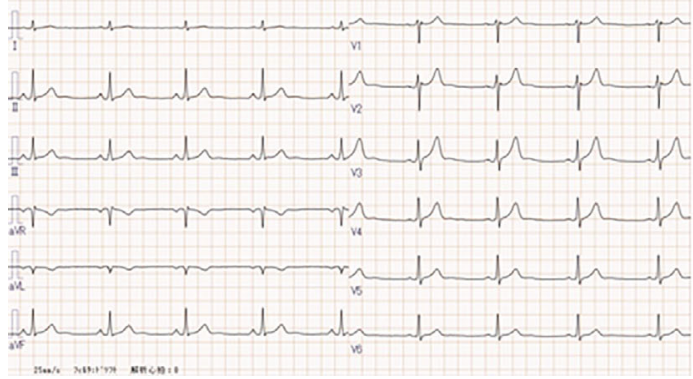

(B)

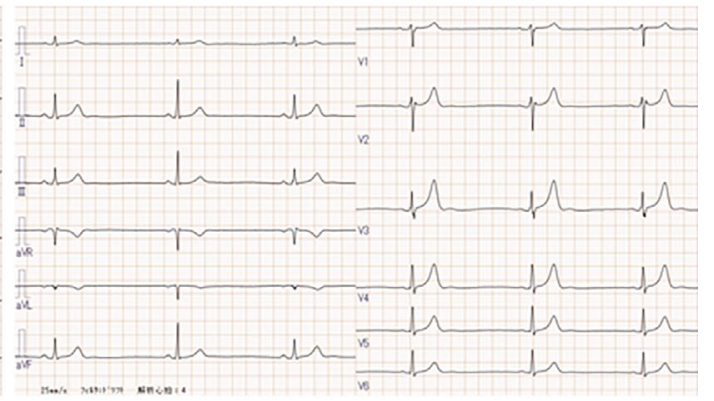

Figure 3. Serial electrocardiograms (ECGs) of the patient with SFT/HPC before and during pazopanib treatment. (A) An ECG before pazopanib treatment showing a heart rate of $50 \mathrm{bpm}$. (B) An ECG at admission during pazopanib treatment, showing sinus bradycardia of $34 \mathrm{bpm}$, a normal QRS complex, and a normal QTc of $403 \mathrm{~ms}$. (C) An ECG after the short cessation of pazopanib. The patient's heart rate returned to $50 \mathrm{bpm}$ and his fatigue was improved.

pazopanib, neither progression of the metastatic tumors nor recurrence of bradycardia was observed.

\section{Discussion}

We herein report a case of severe symptomatic bradycardia possibly associated with pazopanib administration for SFT/HPC. SFT/HPC is a fibroblastic mesenchymal tumor that often displays a rich branching vasculature, characterized by the formation of endothelial tubes and sprouts with a surrounding sheath of rounded cells $(5,6)$. Pazopanib has been approved for the treatment of patients with vasculaturerich solid tumors, such as renal cell carcinoma and SFT/ HPC, who develop local recurrence or distant metastasis. In addition, several studies have recently reported that angiogenesis inhibitors, including pazopanib, can achieve longlasting SFT/HPC stabilization $(7,8)$. Recent studies have also suggested that pazopanib is effective for treating metastatic SFT/HPC $(7,9,10)$. Based on these reports, pazopanib was initiated for our patient with SFT/HPC at the regular 
(A)

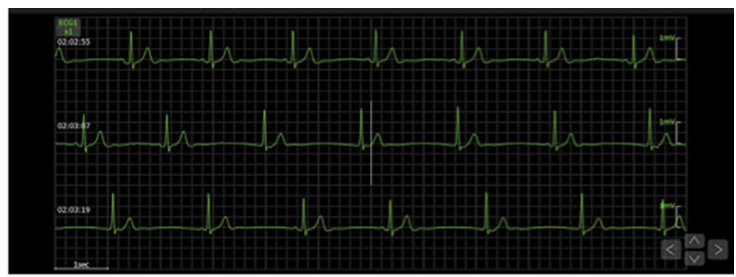

(B)

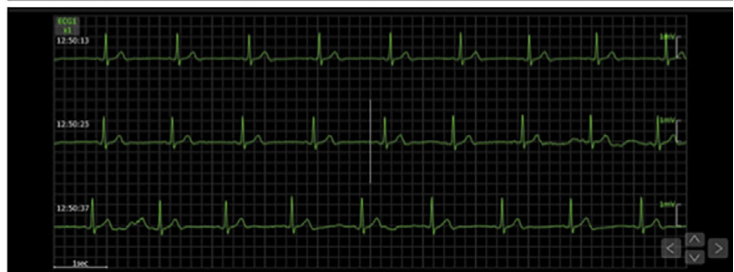

Figure 4. Electrocardiographic monitoring at rest and during exertion. (A) An ECG at rest showing sinus bradycardia of $36 \mathrm{bpm}$. (B) The heart rate increased to $45 \mathrm{bpm}$ on exertion.

dose.

Angiogenesis inhibitors have been shown to be associated with vascular events, such as hypertension, thromboembolism, and rarely aortic dissection (11), as well as cardiac events, such as cardiac dysfunction $(11,12)$. Frequent adverse events related to pazopanib in a phase III trial of SFT/ HPC were diarrhea, fatigue, nausea, and hypertension (2). On the package insert of VOTRIENT ${ }^{\circledR}$, hypertension, cardiac dysfunction, arterial and venous thromboembolism, QT prolongation, and bleeding are indicated as severe adverse events associated with the cardiovascular system. However, only asymptomatic, not symptomatic, bradycardia has been reported, and even then only in $<5 \%$ of cases treated with VOTRIENT $^{\circledR}$. To our knowledge, this is the first report of a patient with symptomatic bradycardia due to pazopanib.

Sinus bradycardia occurs as a pathologic response in a variety of conditions. However, this patient was a young adult man with no cardiovascular comorbidity in his background. Although exercise stress testing or electrophysiologic studies were not performed, we considered it likely that his sinus bradycardia was due to pazopanib-induced dysfunction of the autonomic nervous system. Although the patient had had a relatively slow heart rate before treatment (Fig. 3A), QT prolongation, electrolytic abnormality, and the administration of proarrhythmic drugs did not occur during pazopanib treatment, suggesting a close association between pazopanib and the occurrence of severe bradycardia.

The present patient received GKRS four years before the onset of sinus bradycardia. GKRS focuses intense beams of gamma rays with pinpoint accuracy to treat lesions in the brain. Adverse events of GKRS vary depending on various factors, such as the tumor type, dose delivered, and location of the treated lesion. Reported adverse events of GKRS have included fatigue, nausea, headaches, dizziness or vertigo, hair loss, and localized brain swelling. Most of these effects tend to be mild and spontaneously resolve within a few days after treatment (13). For this reason, it is unlikely that GKRS was associated with the development of sinus bradycardia four years after irradiation.
The VOTRIENT $^{\circledR}$ insert indicates that that half-life of pazopanib $800 \mathrm{mg}$ is $37.8 \mathrm{~h}$, which is consistent with his clinical course. The blood concentration of pazopanib may be associated with the onset of bradycardia. A quarter dose of pazopanib $(200 \mathrm{mg} / \mathrm{day})$ after a short interruption extended its efficacy to some extent, inhibiting SFT/HPC progression for 6 months without proarrhythmic effects. When regular doses of pazopanib are required, implantation of a pacemaker to prevent symptomatic bradycardia will be considered. For the optimal balance of efficacy and safety, basic adverse events, including abnormal heart rates, should be monitored during clinical pazopanib treatment.

Among TKIs, crizotinib and ceritinib, which are smallmolecule TKIs commonly targeting anaplastic lymphoma kinase ALK and ROS proto-oncogene 1, have been reported as associated with bradycardia (14). Pazopanib is a potent multi-targeted TKI that inhibits angiogenic signaling downstream of vascular endothelial growth factor receptor (VEGFR) types 1, 2, and 3; platelet-derived growth factor receptor (PDGFR) alpha and beta; and KIT protooncogene (1). However, whether or not the inhibition of VEGFR or PDGFR or other off-target effects of pazopanib is associated with bradycardia and autonomic nervous system disruption remains unclear. Further studies are needed to determine the precise molecular mechanisms of pazopanib-induced bradycardia.

In conclusion, we encountered a case of pazopanibinduced severe symptomatic bradycardia. After a short interruption, pazopanib was resumed at a quarter-dose without recurrence of bradycardia or new symptoms during six months of follow-up. Attention should be given to the possibility of bradycardia during pazopanib treatment.

The authors state that they have no Conflict of Interest (COI).

\section{References}

1. Hamberg P, Verweij J, Sleijfer S. (Pre-) clinical pharmacology and activity of pazopanib, a novel multikinase angiogenesis inhibitor. Oncologist 15: 539-547, 2010.

2. van der Graaf WT, Blay JY, Chawla SP, et al. Pazopanib for metastatic soft-tissue sarcoma (PALETTE): a randomised, doubleblind, placebo-controlled phase 3 trial. Lancet 379: 1879-1886, 2012.

3. Justice CN, Derbala MH, Baich TM, et al. The Impact of Pazopanib on the Cardiovascular System. J Cardiovasc Pharmacol Ther 23: 387-398, 2018.

4. US National Cancer Institute. Common Terminology Criteria for Adverse Events, version 5.0. Released November 27, 2017.

5. Stout AP, Murray MR. Hemangiopericytoma: A Vascular Tumor Featuring Zimmermann's Pericytes. Ann Surg 116: 26-33, 1942.

6. Louis DN, Perry A, Reifenberger G, et al. The 2016 World Health Organization Classification of Tumors of the Central Nervous System: a summary. Acta Neuropathol 131: 803-820, 2016.

7. Lee SJ, Kim ST, Park SH, et al. Successful use of pazopanib for treatment of refractory metastatic hemangiopericytoma. Clin Sarcoma Res 4: 13, 2014.

8. Park MS, Patel SR, Ludwig JA, et al. Activity of temozolomide and bevacizumab in the treatment of locally advanced, recurrent, 
and metastatic hemangiopericytoma and malignant solitary fibrous tumor. Cancer 117: 4939-4947, 2011.

9. Kasper B, Sleijfer S, Litière S, et al. Long-term responders and survivors on pazopanib for advanced soft tissue sarcomas: subanalysis of two European Organisation for Research and Treatment of Cancer (EORTC) clinical trials 62043 and 62072. Ann Oncol 25: 719-724, 2014.

10. Sleijfer S, Ray-Coquard I, Papai Z, et al. Pazopanib, a multikinase angiogenesis inhibitor, in patients with relapsed or refractory advanced soft tissue sarcoma: a phase II study from the European organisation for research and treatment of cancer-soft tissue and bone sarcoma group (EORTC study 62043). J Clin Oncol 27: 3126-3132, 2009.

11. Takada M, Yasui $\mathrm{T}$, Oka $\mathrm{T}$, et al. Aortic dissection and cardiac dysfunction emerged coincidentally during the long-term treatment with angiogenesis inhibitors for metastatic renal cell carcinoma.
Int Heart J 59: 1174-1179, 2018.

12. Nazer B, Humphreys BD, Moslehi J. Effects of novel angiogenesis inhibitors for the treatment of cancer on the cardiovascular system: focus on hypertension. Circulation 124: 1687-1691, 2011.

13. Werner-Wasik M, Rudoler S, Preston PE, et al. Immediate side effects of stereotactic radiotherapy and radiosurgery. Int J Radiat Oncol Biol Phys 43: 299-304, 1999.

14. Ou SH, Tang Y, Polli A, Wilner KD, Schnell P. Factors associated with sinus bradycardia during crizotinib treatment: a retrospective analysis of two large-scale multinational trials (PROFILE 1005 and 1007). Cancer Med 5: 617-622, 2016.

The Internal Medicine is an Open Access journal distributed under the Creative Commons Attribution-NonCommercial-NoDerivatives 4.0 International License. To view the details of this license, please visit (https://creativecommons.org/licenses/ by-nc-nd/4.0/).

\section{(C) The Japanese Society of Internal Medicine Intern Med Advance Publication}

